\title{
Calculation of surface diffusivity and residence time by molecular dynamics with application to nanoscale selective-area growth
}

\author{
S. Almeida ${ }^{1 *}$, E. Ochoa ${ }^{1}$, J.J. Chavez ${ }^{1}$, X. W. Zhou ${ }^{2}$, and D. Zubia ${ }^{1}$ \\ ${ }^{1}$ University of Texas at El Paso, Department of Electrical and Computer Engineering, El Paso, Texas \\ 79968, USA \\ ${ }^{2}$ Sandia National Laboratories, Mechanics of Materials Department, Livermore, California 94550, USA \\ *sfalmeida@utep.edu; phone: (915)747-6580
}

\begin{abstract}
The surface diffusivity and residence time were calculated by molecular dynamics simulations in order to solve the surface diffusion equations for selective-area growth. The calculations for $\mathrm{CdTe} / \mathrm{CdS}$ material system were performed in substrates with $\mathrm{Cd}$ termination and $\mathrm{S}$ termination. The surface diffusivity and residence time were obtained at different temperatures $(600 \mathrm{~K}, 800 \mathrm{~K}, 1000 \mathrm{~K}, 1200 \mathrm{~K}$, and $1400 \mathrm{~K})$. The thermal activation energies were extracted from Arrhenius equation for each substrate termination. Thereafter, values obtained by molecular dynamics were used in a surface diffusion model to calculate the surface concentration profile of adatoms. Alternating the surface termination has the potential to achieve nanoscale selective-area growth without the need of a dielectric film as a mask.
\end{abstract}

Keywords: A1. Surface diffusivity, A1. Residence time, A3. Surface diffusion, A3. Selective-area growth, B1. Cadmium telluride

\section{Introduction}

Selective-area growth (SAG) is widely used to create micro and nano-structures of a variety of compound semiconductors, including InP(Lu, Tran, Ko, N, Chen, \& Chang-Hasnain, 2012), CdTe(Terrazas, et al., 2005), GaAs (Noborisaka, Motohisa, \& Fukui, 2005), GaN (Wang, et al., Growth kinetics and mass transport mechanisms of GaN columns by selective area metal organic vapor phase epitaxy, 2014), InGaAs (Coleman, Lammert, Osowski, \& Jones, 1997), within others. In this bottom-up fabrication technique a material structure is grown on a patterned substrate usually by metalorganic chemical vapor deposition (MOCVD)(Coltrin \& Mitchell, Mass transport and kinetic limitations in MOCVD selective-area growth, 2003), metalorganic vapor-phase epitaxy (MOVPE)(Wullner, Chahoud, Schrimpf, Bonsch, Wehmann, \& Schlachetzki, 1999), molecular beam epitaxy (MBE) (Heib, Riedlberger, Spirkoska, Bichler, Abstreiter, \& Morral, 2008), or close space sublimation (CSS)(Kiriya, et al., 2012). SAG has the potential to integrate III-V materials on silicon closing the gap between optoelectronic devices and CMOS circuits(Ren, Ng, Li, Sun, \& Chang-Hasnain, 2013). Furthermore, nanoscale selectivearea growth is a promising option to reduce the interfacial defect density in lattice mismatched materials such as CdTe/CdS solar cells due to strain partitioning and three-dimensional stress relief (nanoheteroepitaxy) (Zubia, et al., 2007). Recently, Aguirre et al. reported the growth of 
CdTe single grains on CdS via nanoscale selective-area growth(Aguirre, et al., 2014). However the growth dynamics of nanoscale selective-area growth are not well understood.

There are two predominant models which describe the mechanisms for SAG, vapor diffusion(Coltrin, Hsu, Scrymgeour, Creighton, Simmons, \& Matzke, 2008; Coronell \& Jensen, 1991) and surface diffusion(Yamaguchi, Ogasawara, \& Okamoto, 1992; Greenspanan, Blaauw, Emmerstorfer, Glew, \& Shih, 2003). In the vapor diffusion model, the deposition reaction is characterized by a sticking coefficient which is assumed much higher on window openings than on masked areas. This occurs by allowing re-evaporation of adatoms arriving to the mask while ignoring surface migration on the mask and window regions (Jones, Osowski, Lammert, Dantzig, \& Coleman, 1995). In this scenario mass transport is dominated by the change of the vapor concentration. The result is accurate modeling of growth selectivity when the feature size of the mask is larger than $\sim 10 \mu \mathrm{m}$ (Ujiharaa, Yoshidaa, Leea, \& Takeda, 2006). In contrast, the surface diffusion model considers the surface migration of adatoms on the mask and window regions. The surface diffusivities of the adatoms on the mask and window surfaces define the change of the surface concentration. In this case, growth selectivity is modelled well when the feature size is small (below $\sim 10 \mu \mathrm{m}$ )(Ujiharaa, Yoshidaa, Leea, \& Takeda, 2006). The principal parameters that define the surface diffusion model are the surface diffusivity $\left(D_{s}\right)$, diffusion length $\left(\lambda_{s}\right)$, and residence time $\left(\tau_{s}\right)$ of the adatoms. However in most reported experiments, these parameters are obtained empirically (Yamaguchi, Ogasawara, \& Okamoto, 1992; Greenspanan, Blaauw, Emmerstorfer, Glew, \& Shih, 2003; Fujii, Ekawa, \& Yamazaki, 1995). Yamaguchi et al. obtained the diffusion length by depositing $\mathrm{GaAs}$ on patterned $\mathrm{SiO}_{2}$ and $\mathrm{W}$ masks and measuring the width of the deposition-free region at the mask edges (Yamaguchi, Ogasawara, \& Okamoto, 1992). Fujii et al. calculated the surface parameters of InP on InP(001) substrates with $\mathrm{SiO}_{2}$ mask by fitting the theoretical model to the growth rate (Fujii, Ekawa, \& Yamazaki, 1995). A similar methodology was employed by Greenspan et al(Greenspanan, Blaauw, Emmerstorfer, Glew, \& Shih, 2003). Even though Swartzentruber measured the surface diffusion directly using a scanning tunneling microscope (Swartzentruber, 1996) it is still complicated to measure these parameters in most deposition systems.

In this work, the surface diffusivity and residence time of the $\mathrm{CdTe} / \mathrm{CdS}$ material system are calculated using high fidelity molecular dynamics (MD) simulations in order to provide insight into the growth dynamics for nanoscale selective-area growth in the surface-diffusion regime. Thereafter, the MD values were applied to solve the surface diffusion model under CSS conditions. A surface concentration profile was obtained using different substrate terminations showing the potential to achieve nanoscale selective-area growth with differential atomic surface termination.

\section{Molecular dynamics simulations}

The MD simulations were performed using the Large-Scale Atomic/Molecular Massively Parallel Simulator (LAMMPS) code (Plimpton, 19951). The CdTe/CdS material system was 
selected due to its technological interest and the availability of a validated Stillinger-Weber potential (Zhou, Ward, Martin, Swol, Cruz-Campa, \& Zubia, 2013). However this methodology can be adapted to other material systems by using the appropriate interaction potentials. Surface diffusivities and residence times were calculated involving the simulation of $\mathrm{Cd}$ and Te adatom migration on $\mathrm{Cd}$ - or S-terminated $\mathrm{CdS}(0001)$ substrates. Moreover each situation was simulated at temperatures of $600 \mathrm{~K}, 800 \mathrm{~K}, 1000 \mathrm{~K}, 1200 \mathrm{~K}$, and $1400 \mathrm{~K}$. Periodic boundary conditions were used in the 10 distinct simulations.

\subsection{Surface diffusivity calculations}

The computational substrate was created by positioning atoms according to the crystal structure, equilibrium lattice constant, and crystallographic orientation of a wurtzite crystal with a top surface oriented in the [0001]. Each CdS single crystal substrate used consisted of 60 [11-20], 40 [-1100], and 31 [0001] planes in the $x, y$, and $z$ directions respectively, as shown in Figure 1. The lattice parameters for the wurtzite crystal were $a=4.12597 \AA$ and $c=6.73768 \AA$.

An initial substrate temperature was introduced by assigning atom velocities using a Gaussian distribution. Periodic boundary conditions were employed in the horizontal directions ( $x$ and $y)$, and a free boundary condition in the vertical $z$ direction. To prevent crystal drift due to adatom momentum transfer the substrate atoms of the bottom two monolayers were fixed by setting the velocity of these atoms to zero throughout the simulation. An intermediate region starting at the top of the fixed bottom monolayers and ending four monolayers below the substrate's top surface was maintained at a desired substrate temperature using a NoseHoover (Hoover, 1985) algorithm in order to approximate isothermal growth conditions while maintaining a surface that realistically captures interactions in this area. The adatom vapor consisted of $100 \mathrm{Cd}$ and 200 Te atoms that were randomly placed $50 \AA$ above the substrate's surface. The Te/Cd ratio of 2 was selected in order to maintain stoichiometric conditions in vapor phase (Alamri, 2003). Cd and Te atoms were set with an initial velocity of $-8 \AA / p s$ directed along the $\mathrm{z}$ direction to simulate the drift velocity of $\mathrm{Cd}$ and Te atoms in a He atmosphere. $\mathrm{A}$ reflective wall that redirected evaporated atoms back to the substrate was placed $10 \AA$ above the surface using the LAMMPS' fix wall/reflect command. Figure 1(a) and (b) show the schematic of the MD simulations with Cd-terminated and S-terminated substrates, respectively. 

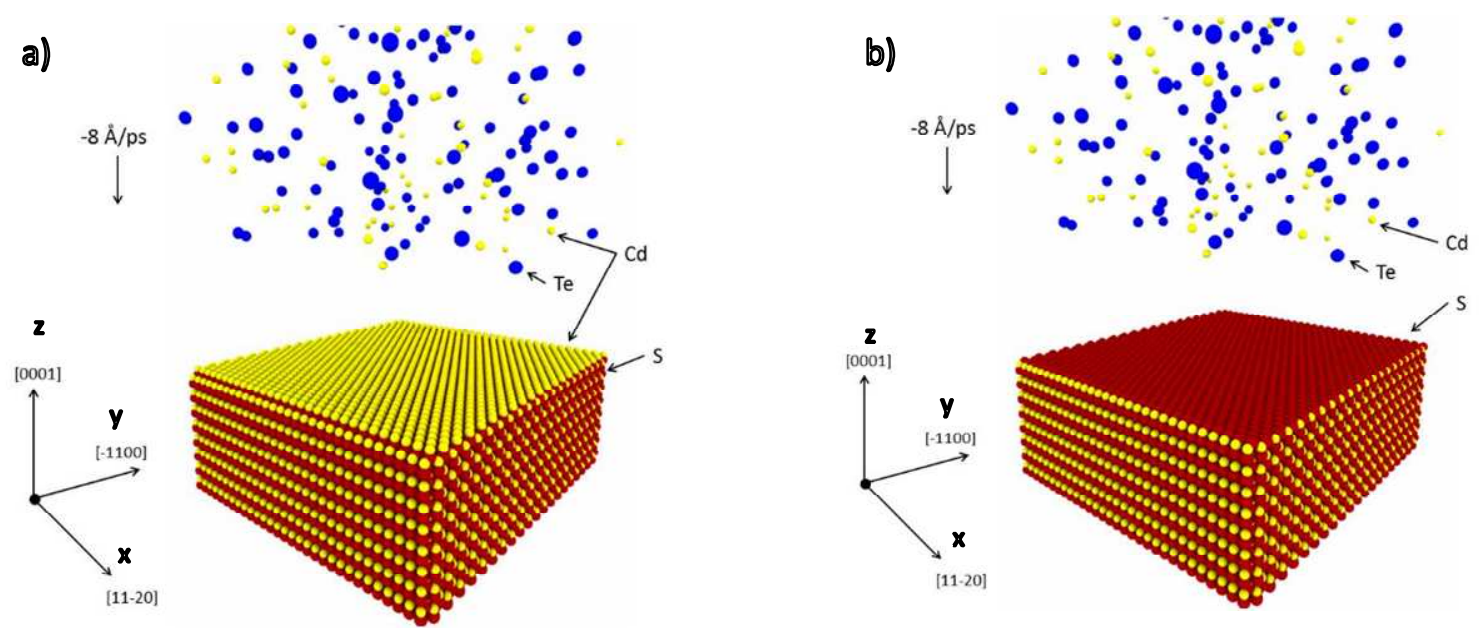

Figure 1. Schematic of the molecular dynamic simulations with a) Cd-terminated and b) Sterminated substrates. Images created with OVITO (Stukowski, 2010).

A time step of 0.004 ps was employed for a total simulation time of 840 ps. The first 40 ps were ignored for allowing $\mathrm{Cd}$ and Te atoms to arrive at the substrate surface. Thereafter, the mean-square displacements (MSD) in the $x$ and $y$ directions were computed for atoms found within a region between the substrate surface and $10 \AA$ above using the simulator's compute command. The calculations were performed during the remaining 800 ps ignoring the atoms outside of the MSD region. Figure 2 (a)-(d) plots the MSD values for $\mathrm{Cd}$ and Te adatoms on $\mathrm{Cd}$ terminated and S-terminated $\mathrm{CdS}(0001)$ substrates. over the range of deposition temperatures. In general the MSD increases with temperature. It is also observed that the MSDs are longer on the Cd-terminated CdS compared to the S-terminated CdS. These results indicate that surface termination can influence adatom migration and can potentially be used to achieve selectivearea growth particularly at the nanoscale since growth selectivity is usually achieved by differential surface migration(Wang, et al., Growth kinetics and mass transport mechanisms of GaN columns by selective area metal organic vapor phase epitaxy, 2014).
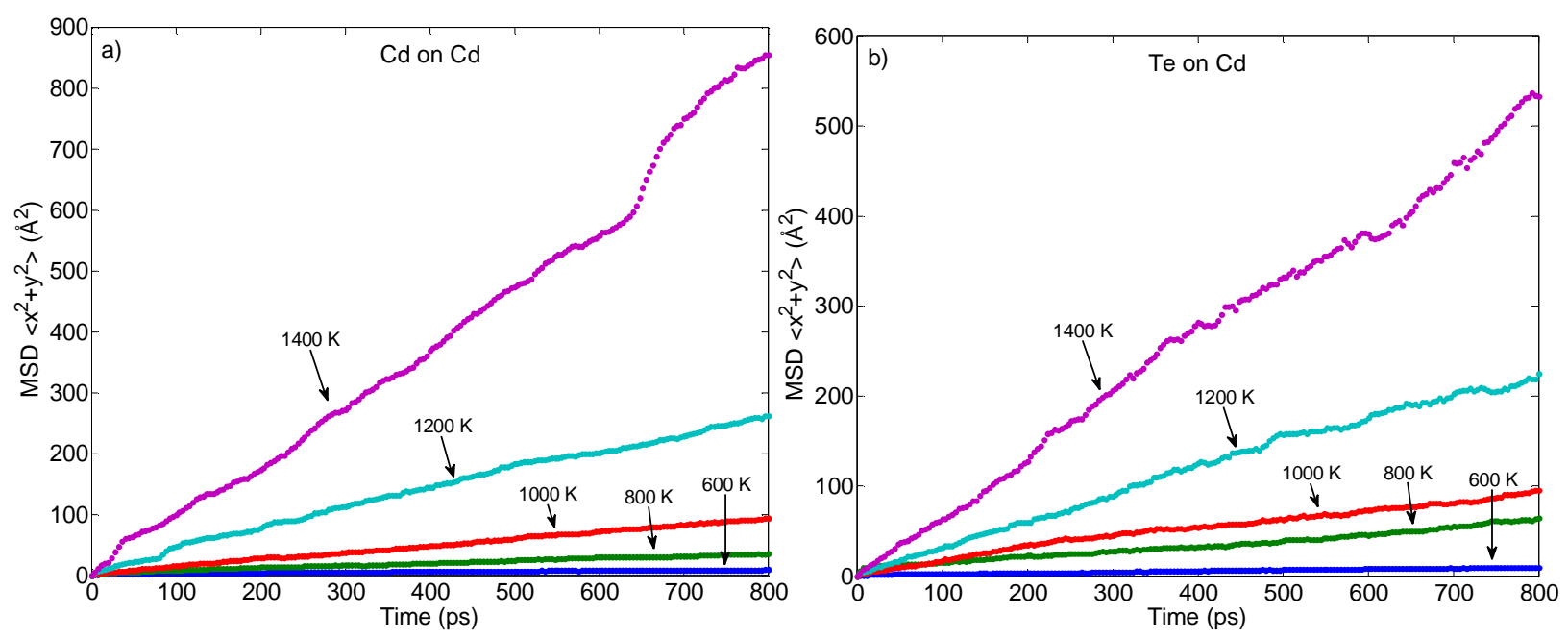

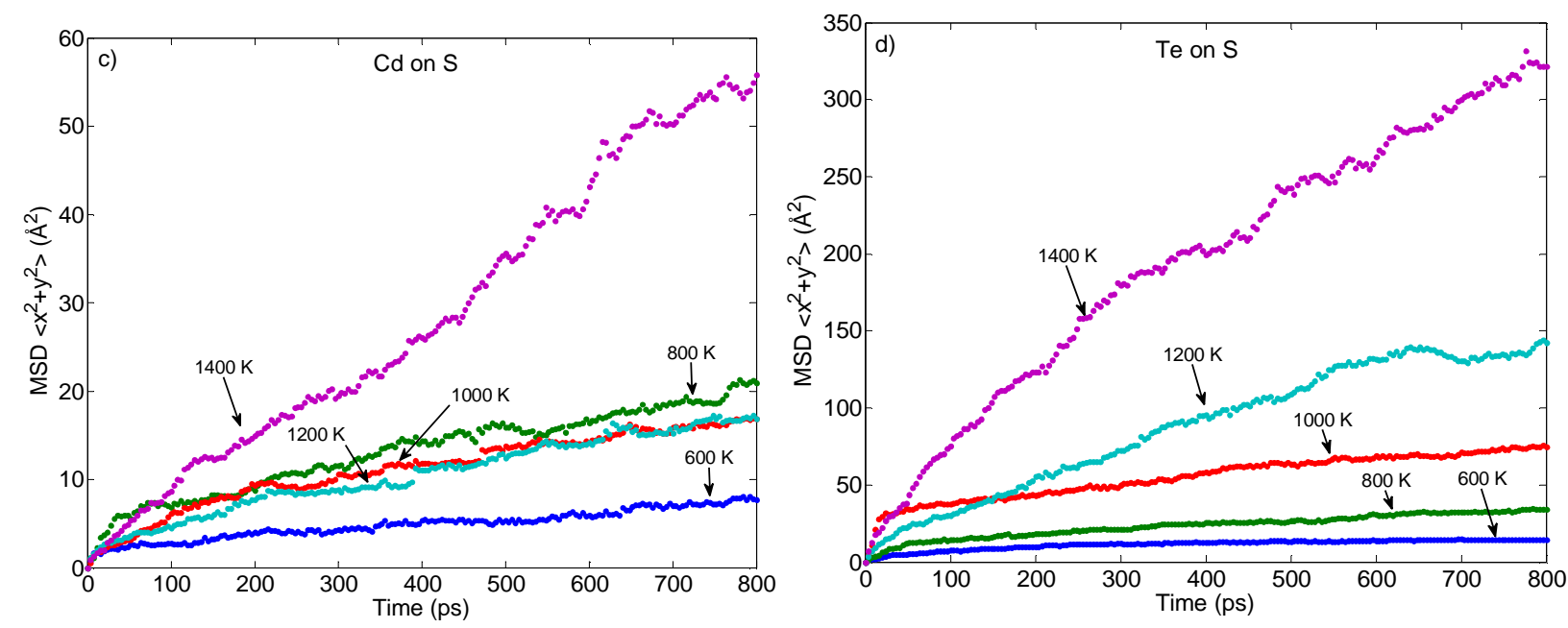

Figure 2. Mean square displacement of adatom on the surface at different temperatures for; (a) $\mathrm{Cd}$ and (b) Te adatoms on Cd-terminated CdS, and (c) Cd and (d) Te adatoms on S-terminated CdS.

The surface diffusivity was calculated from the MSD data using the Einstein relation

$$
D_{s}=\frac{1}{2 d} \lim _{t \rightarrow \infty} \frac{d}{d t}(M S D(t))
$$

where $d$ is the number of dimensions of motion. In this case $d=2$. Over time the MSD becomes linear due to the diffusion process (Dobbs \& Doren, 1992) and the surface diffusivities were calculated by curve fitting the MSD data to the linear portion of the graph. Table 1 contains the calculated surface diffusivities of the four situations at different temperatures.

Table 1. Calculated surface diffusivities for different temperatures and substrate terminations.

\begin{tabular}{|c|c|c|c|c|}
\hline Temperature (K) & Cd on $\mathbf{C d ~}\left(\mathrm{cm}^{\mathbf{2}} / \mathbf{s}\right)$ & Cd on S $\left(\mathrm{cm}^{2} / \mathbf{s}\right)$ & Te on $\mathbf{C d ~}\left(\mathrm{cm}^{2} / \mathbf{s}\right)$ & Te on S $\left(\mathrm{cm}^{2} / \mathbf{s}\right)$ \\
\hline $\mathbf{6 0 0}$ & $2.0555 \times 10^{-7}$ & $1.7440 \times 10^{-7}$ & $2.6725 \times 10^{-7}$ & $2.1945 \times 10^{-7}$ \\
\hline $\mathbf{8 0 0}$ & $9.6625 \times 10^{-7}$ & $4.6925 \times 10^{-7}$ & $1.6400 \times 10^{-6}$ & $7.1850 \times 10^{-7}$ \\
\hline $\mathbf{1 0 0 0}$ & $2.8350 \times 10^{-6}$ & $3.5925 \times 10^{-7}$ & $2.6725 \times 10^{-6}$ & $1.3995 \times 10^{-6}$ \\
\hline $\mathbf{1 2 0 0}$ & $7.8975 \times 10^{-6}$ & $4.4200 \times 10^{-7}$ & $7.0125 \times 10^{-6}$ & $4.3725 \times 10^{-6}$ \\
\hline $\mathbf{1 4 0 0}$ & $2.6100 \times 10^{-5}$ & $1.7568 \times 10^{-6}$ & $1.6133 \times 10^{-5}$ & $9.3150 \times 10^{-6}$ \\
\hline
\end{tabular}

On a $\mathrm{CdS}(0001)$ plane atoms jump in hexagonal paths with equal probabilities, therefore the surface diffusivity can be isotropic in any directions. From the plane symmetry it can be expected that a jumping atom would fall (with the maximum probability) a distance $R$ away from the origin point after many jumps but the angular distribution of the final position can be uniform.

The surface diffusivity can be expressed by the Arrhenius equation, 


$$
D_{s}=\operatorname{Aexp}\left(-\frac{Q}{k_{B} T}\right)
$$

where $A$ is a material constant, $Q$ is the thermal activation energy, $k_{B}$ is the Boltzmann constant, and $T$ is the temperature. Figure 3 shows the relationship between $\ln (D s)$ and 1000/T agreeing with the Arrhenius behavior. The activation energies of the four cases were calculated from Figure 3 and are shown in Table 2. We noted that the activation energies calculated for Te adatoms on the $\mathrm{Cd}$-and S-terminated CdS $(0.350 \mathrm{eV}$ and $0.327 \mathrm{eV}$, respectively) agree well with the experimental value reported by Castro-Rodriguez, et al. (0.332 eV) (Castro-Rodrigueza, Martel, Mendez-Gamboa, \& Pena, 2007). The activation energies were higher for adatoms migrating on surfaces terminated with atoms from the same group as the adatoms, e.g., group II on group II, or group VI on group VI (Cd on Cd and Te on S). In contrast, adatoms migrating on surfaces terminated with atoms from a dissimilar group had the lowest activation energies (Te on $\mathrm{Cd}$ and $\mathrm{Cd}$ on $\mathrm{S}$ ).

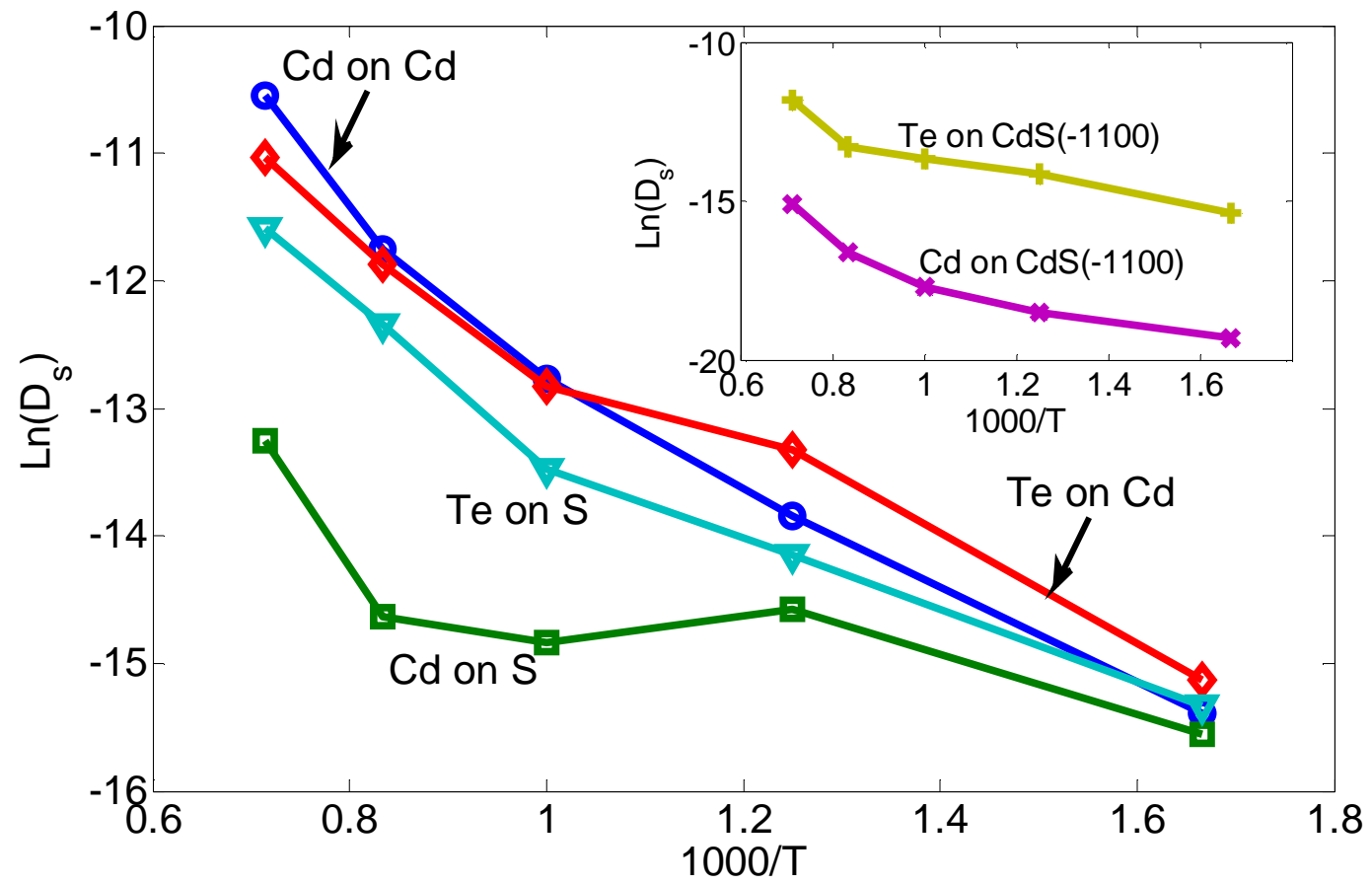

Figure 3. Arrhenius plot for $\mathrm{Cd}$ and Te adatoms on $\mathrm{Cd}$ and $\mathrm{S}$ substrate terminations for $\mathrm{CdS}(0001)$. The inset shows the surface diffusivities for $\mathrm{Cd}$ and Te atoms on CdS(-1100).

Table 2. Activation energies $\mathrm{Q}(\mathrm{eV})$ for $\mathrm{Cd}$ and Te surface diffusion on $\mathrm{Cd}$ - and S-terminated CdS.

\begin{tabular}{|c|c|c|c|}
\hline Cd on Cd & Te on Cd & Cd on S & Te on S \\
\hline 0.418 & 0.350 & 0.154 & 0.327 \\
\hline
\end{tabular}

The crystal orientation of the substrate also plays an important role in SAG due to different surface diffusivities (Greenspanan, Blaauw, Emmerstorfer, Glew, \& Shih, 2003). To analyze this effect, MD simulations were performed for $\mathrm{CdS}(-1100)$ substrates using the same settings mentioned above. It is important to notice that the termination for this orientation is irrelevant due to the equal amount of $\mathrm{Cd}$ and Te atoms in the surface. In this case the surface 
diffusivity is anisotropic along the surface plane directions therefore the calculated diffusivities is the average of all directions on that plane. The inset in Figure 3 shows the Arrhenius plot for $\mathrm{Cd}$ and Te surface diffusivities average along CdS(-1100) substrates. The calculated activation energies for $\mathrm{Cd}$ and $\mathrm{Te}$ adatoms are $0.346 \mathrm{eV}$ and $0.280 \mathrm{eV}$, respectively. It is possible to observe different surface diffusivities depending on substrate orientation as expected due to different surface energies.

\subsection{Residence time calculations}

The residence time calculation consisted of simulating a single adatom deposited on the $\mathrm{CdS}(0001)$ surface and measuring the time required to desorb the adatom. In order to determine when the adatom had first bonded to the substrate and later desorbed, its coordination number was tracked throughout the simulation using LAMMPS's compute coord command. The coordination number was defined as the number of $\mathrm{Cd}$ and $\mathrm{S}$ neighbor atoms found within a specified cutoff distance ( $4 \AA$ was used as this number is slightly larger than the CdTe closest neighbor interatomic spacing). Under these conditions, the adatom in vapor phase would have a coordination number of zero, and a coordination number greater than zero would mean that the adatom is bonded on the substrate surface. The residence time was then found by measuring the amount of time the adatom was bonded before desorbing. A total simulation time of 20,000 ps was employed for the Te adatom on S-terminated substrate and Cd adatom on Cd-terminated substrate cases. During this interval the average residence time for each temperature was calculated. The $\mathrm{Cd}$ adatom on S-terminated substrate and Te adatom on Cdterminated substrate cases required a longer simulation time of 200,000 ps because their residence time were larger. The substrate configuration used for these simulations was the same as described in section 2.1. for $\mathrm{CdS}(0001)$. Figure 4 plots the recorded residence times for the 4 cases over a range of temperatures. It is interesting to note that the residence time, similar to the surface diffusivity, is influenced by the temperature and the substrate termination and can potentially be used for selective-area growth. Residence times for the $\mathrm{Cd}$ adatom on S-terminated substrate and Te adatom on Cd-terminated cases at $600 \mathrm{~K}$ and $800 \mathrm{~K}$ temperatures were not captured within the simulated time and are therefore assumed to be longer than 200,000 ps. In the following section, the substrate termination was used to achieve differential surface migration and residence time in a patterned substrate and calculate the surface concentration profile of the adatoms. 


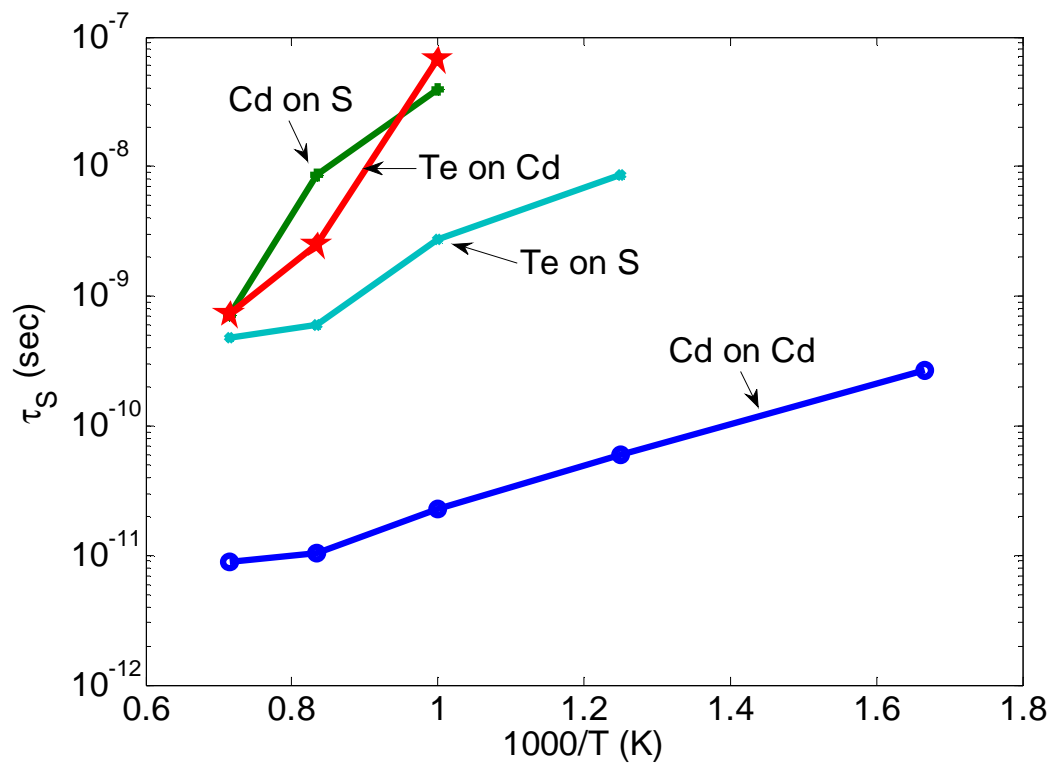

Figure 4. Plot of residence times recorded for all case studies over a range of temperatures.

\section{Surface diffusion model}

In this section, the values of $D_{s}$ and $\tau_{s}$ obtained from MD simulations on CdS(0001) were used to obtain the surface concentration profile of adatoms under CSS deposition conditions. In this deposition technique, CdTe is deposited under a He atmosphere and the substrate and source temperatures can be manipulated independently. Due to the small separation between the source and the substrate (typically 1 to $4 \mathrm{~mm}$ ), it can be assumed that mass transport takes place in the stagnant layer above the substrate surface. In this zone the reactant species obeys Fick's law (Cruz-Campa \& Zubia, 2009) as shown in Figure 5(a). Under steady-state, the vapor phase flux density $\left(\underline{J}_{v}\right)$ of the reactant species in the stagnant layer can be expressed as,

$$
J_{V}=J_{d}-J_{e}
$$

where $J_{d}$ is the flux density of the atoms drifting to the surface, which depends on the mean free path $(L)$ and the vapor concentration of the reactant species (Yamaguchi, Ogasawara, \& Okamoto, 1992). $J_{e}$ is the flux density of the re-evaporated atoms from the surface and depends on the surface concentration $\left(N_{s}\right)$ and $\tau_{s}$ of the adatoms on the surface as follows,

$$
J_{e}=\frac{N_{s}}{\tau_{s}}
$$

Under the assumption of a linear gradient, $J_{v}$ can also be expressed as,

$$
J_{v}=D_{V}^{H e} \frac{N_{v}-N_{L}}{h-L}
$$

where $D_{V}^{H e}$ is the diffusion coefficient of $\mathrm{Cd}$ or Te atoms in a He atmosphere, $h$ is the stagnant layer thickness, $N_{v}$ is the vapor concentration of $\mathrm{Cd}$ or Te at $h$, and $N_{L}$ is the vapor concentration at $L$. $L$ depends on the source temperature $(T)$, the pressure $(P)$, and the molecular diameter 
$\left(d_{m}\right)$ of Cd or Te (2.606 and $3.966 \AA$ respectively (Alamri, 2003)) and is expressed by (Alamri, 2003),

$$
L=\frac{k_{B} T}{\sqrt{2} \pi d_{m}^{2} P}
$$

The drift velocity used in the MD calculations was calculated as $D_{V}^{H e} / L . D_{V}^{H e}$ can be calculated using the Stefan-Boltzmann model elaborated in (Cruz-Campa \& Zubia, 2009) $\left(23.596 \mathrm{~cm}^{2} / \mathrm{s}\right.$ for $\mathrm{Cd}$ and $16.908 \mathrm{~cm}^{2} / \mathrm{s}$ for Te at $1200 \mathrm{~K}$ with a pressure of $30 \mathrm{Torr}$ ). Similar to $J_{v}$ under the assumption of a linear gradient, $J_{d}$ can be expressed as follows,

$$
J_{d}=D_{V}^{H e} \frac{N_{L}}{L}
$$

Mass conservation also takes place on the surface and the continuity equation under steady-state conditions can be expressed by,

$$
J_{d}-J_{e}-\nabla J_{L}-\nabla J_{S}=0
$$

where $J_{L}$ is the lateral flux density due to the concentration gradient in the vapor-phase (Wullner, Chahoud, Schrimpf, Bonsch, Wehmann, \& Schlachetzki, 1999). This is given by,

$$
J_{L}=D_{V}^{H e} \nabla N_{s}
$$

However, for a nano scale region the net lateral flux can be considered to equal zero due to the relatively large mean free path $(L)$ of $\mathrm{Cd}$ or Te in He $(4.442 \mu \mathrm{m}$ and $1.335 \mu \mathrm{m}$ respectively at $1200 \mathrm{~K}$ with a pressure of 30 Torr). Moreover $\nabla J_{L}$ is spatially uniform since any information that was encoded at the nana scale is lost over a large distance. This is depicted in Figure 5(a) where $\mathrm{Cd}$ and Te atoms arrive and leave from one nano-scale region to another, causing the net lateral flux to be zero. Thus for nano-scale SAG the surface flux dominates (Ujiharaa, Yoshidaa, Leea, \& Takeda, 2006) and equation (8) can be expressed as,

$$
J_{d}-J_{e}=\nabla J_{s}
$$

$J_{s}$ is the flux density of the adatoms on the substrate surface. $J_{s}$ is proportional to $D_{s}$ and can be expressed as,

$$
J_{s}=D_{s} \nabla N_{s}
$$

$D_{s}$ can take different values for the mask and window areas in selective-area growth as shown in Figure 5(b). Moreover $D_{s}$ can be different along $\mathrm{x}$ and $\mathrm{y}$ directions, however for $\mathrm{Cd}(0001)$ substrates $D_{s}$ can be considered isotropic due to the plane symmetry. In this case $D_{s}$ can be expressed as a piecewise function as follows:

$$
D_{s}=\left\{\begin{array}{cc}
D_{\text {Window }} & \text { Window Area } \\
D_{\text {Mask }} & \text { Mask Area }
\end{array} .\right.
$$

As well, $\tau_{s}$ can take different values depending on the details of the surface material, 


$$
\tau_{s}=\left\{\begin{array}{cc}
\tau_{\text {Window }} & \text { Window Area } \\
\tau_{\text {Mask }} & \text { Mask Area }
\end{array}\right.
$$

a)

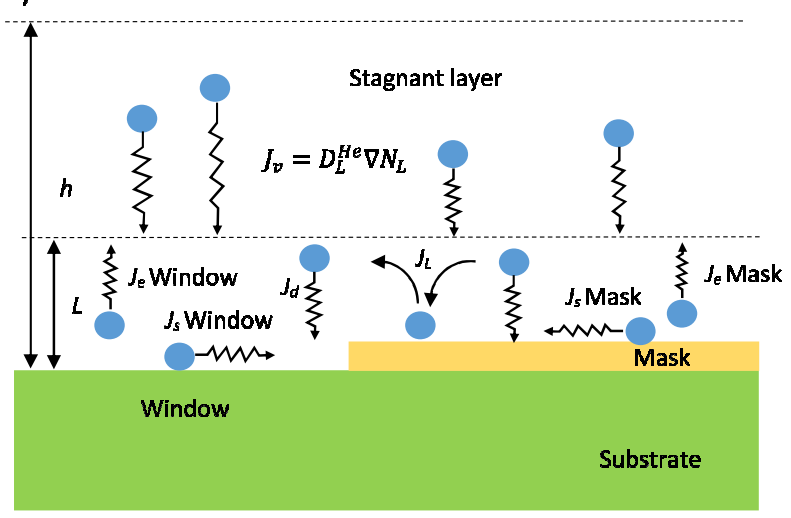

b)

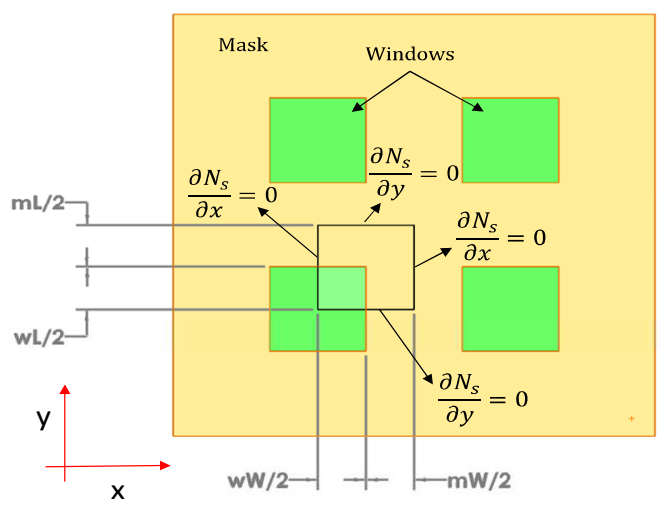

Figure 5. (a) Schematic diagram of the motion of the reactant species in the stagnant layer, $J_{e}$ and $J_{s}$ have distinct values on the mask and window areas. (b) 2D view of the substrate where the continuity equations are solved in the area enclosed by the box under Neumann boundary conditions. $\mathrm{mL}$ indicates mask length, $\mathrm{wL}$ window lengths, $\mathrm{mW}$ mask width, and $\mathrm{wW}$ window width.

Equations 3-7 and 10-13 were solved numerically using Liebmann's method in 2D, assuming that $L$ is larger than the thickness of the mask. Neumann Boundary conditions were set in a periodic area to represent the entire substrate, as shown in the box in Figure 5(b). In this case, the mask area was represented by a S-terminated CdS(0001) substrate and the window area by $\mathrm{Cd}$-terminated $\mathrm{CdS}(0001)$. The values of $D_{s}$ and $\tau_{s}$ at $1200 \mathrm{~K}$ were used to obtain the $N_{s}$ profile for $C d$ and Te atoms in an area of $50 \times 50 \mathrm{~nm}^{2}$.

Figures $6(\mathrm{a})$ and (b) show the $N_{s}$ profile normalized to the maximum concentration for $\mathrm{Cd}$ and Te adatoms, respectively. The dashed lines represent the transition from Cd-termination to S-termination at $25 \mathrm{~nm}$ in the $\mathrm{x}$ and $\mathrm{y}$ directions. For $\mathrm{Cd}$ adatoms the higher concentration occurs on the S-terminated area. $D_{s}$ is one order of magnitude larger, while $\tau_{s}$ is three orders of magnitude smaller on the Cd-terminated compared to the S-terminated. Due to the large difference in $D_{s}$ and $\tau_{s}$, the transition from the minimum $N_{s}$ to the maximum $N_{s}$ markedly occurs over a narrow distance of $8 \mathrm{~nm}$.

In contrast, Te adatoms had a higher concentration on the Cd-terminated area. In this case, Te adatoms have a similar value for $D_{s}$ and only one order of magnitude higher $\tau_{s}$ on the Cd-terminated surface. The transition from the minimum $N_{s}$ to the maximum $N_{s}$ occurs over a distance of $10 \mathrm{~nm}$. $N_{s}$ profiles show that nanoscale selective-area can be achieved using different surface terminations and alternating the evaporation of $\mathrm{Cd}$ and $\mathrm{Te}$ atoms during deposition. 

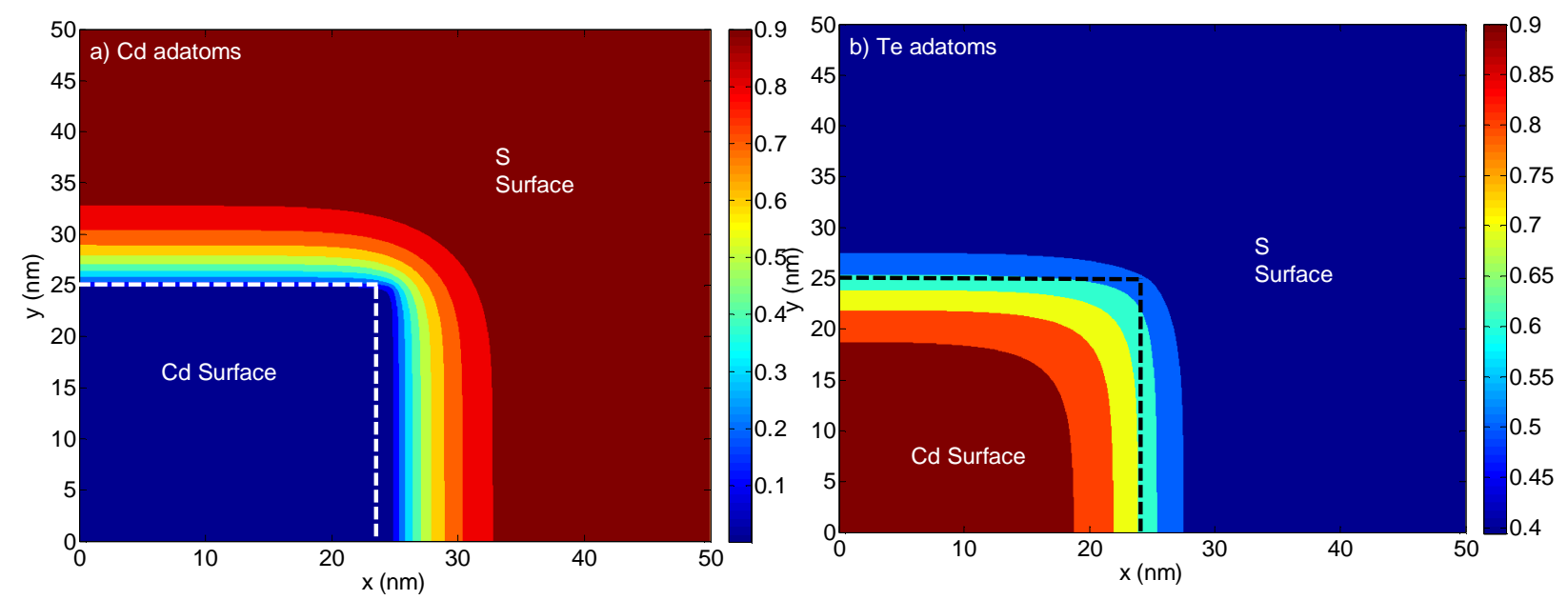

Figure 6. Surface concentration profile for (a) Cd and (b) Te adatoms using the S-terminated substrate as the mask.

\section{Conclusions}

Surface diffusivities and residence times were calculated by MD simulations and then applied to surface diffusion equations to predict nanoscale selective-area growth using the differential atomic substrate termination. The surface diffusivities of $\mathrm{Cd}$ and Te adatoms were calculated using the MSD on Cd-terminated and S-terminated CdS(0001) substrates at different temperatures. The thermal activation energies were extracted from Arrhenius behavior of $\mathrm{Cd}$ and Te surface diffusivities on Cd-terminated and S-terminated substrates. To analyze the effect of crystal orientation, surface diffusivities on S-terminated CdS(-1100) substrates were calculated. Thereafter, the residence times were calculated by monitoring the change in the coordination number of a single $\mathrm{Cd}$ or Te adatom. This parameter showed a high dependence on the temperature and substrate polarity. Finally, the values of surface diffusivity and residence time were used to calculate the surface concentration profile of adatoms under CSS deposition conditions. For $\mathrm{Cd}$ adatoms, the highest concentration occurs in S termination area. Whereas Te adatoms concentrate in $\mathrm{Cd}$ terminated area. $N_{s}$ profiles show that nanoscale selective-area growth can be achieved by alternating the source material and substrate termination eliminating the need to employ patterned dielectric films to accomplish this.

\section{Acknowledgments}

This work was supported in part by NSF through the Center for Energy Efficient Electronics Science (NSF Award 0939514), the PREM program (DMR-1205302), the IGERT program (DGE-0903670), the use of the Virgo2 Cluster (NSF Award CNS-1059430), and Department of Energy through the BRIDGE program (DE-EE0005958).

\section{References}

Aguirre, B. A., Zubia, D., Ordonez, R., Anwar, F., Prieto, H., Sanchez, C. A., et al. (2014). Selective Growth of CdTe on Nano-patterned CdS via Close-Space Sublimation. Journal of ELECTRONIC MATERIALS . 
Alamri, S. N. (2003). The growth of CdTe thin film by close space sublimation system. Physica status solidi (a) , 200 (2), 352-360.

Castro-Rodrıgueza, R., Martel, A., Mendez-Gamboa, J., \& Pena, J. (2007). Nucleation and growth mechanism of CdTe cluster grown on CdS films. Journal of Crystal Growth , 306, 249-253.

Coleman, J. J., Lammert, R. M., Osowski, M. L., \& Jones, A. M. (1997). Progress in InGaAs-GaAs SelectiveArea MOCVD Toward Photonic Integrated Circuits. IEEE Journal of Selected Topics in Quantum Electronics, 3 (3).

Coltrin, M. E., \& Mitchell, C. C. (2003). Mass transport and kinetic limitations in MOCVD selective-area growth. Journal of Crystal Growth , 254, 35-45.

Coltrin, M. E., Hsu, J. W., Scrymgeour, D. A., Creighton, J. R., Simmons, N. C., \& Matzke, C. M. (2008). Chemical kinetics and mass transport effects in solution-based selective-area growth of $\mathrm{ZnO}$ nanorods. Journal of Crystal Growth , 584-593.

Coronell, D. G., \& Jensen, K. F. (1991). Analysis of MOCVD of GaAs on patterned substrates. Journal of Crystal Growth , 114, 581-592.

Cruz-Campa, J. L., \& Zubia, D. (2009). CdTe thin film growth model under CSS conditions. Solar Energy Materials \& Solar Cells , 93, 15-18.

Dobbs, K. D., \& Doren, D. J. (1992). Dynamics of molecular surface diffusion: Origins and consequences of long jumps. The Journal of Chemical Physics, 97 (5), 3722-3735.

Fujii, T., Ekawa, M., \& Yamazaki, S. (1995). A theory for metalorganic vapor phase epitaxial selective growth on planar patterned substrates. Journal of Crystal Growth, 146, 475-481.

Greenspanan, J., Blaauw, C., Emmerstorfer, B., Glew, R., \& Shih, I. (2003). Analysis of a time-dependent supply mechanism in selective area growth by MOCVD. Journal of Crystal Growth, 248, 405-410.

Heib, M., Riedlberger, E., Spirkoska, D., Bichler, M., Abstreiter, G., \& Morral, A. F. (2008). Growth mechanisms and optical properties of GaAs-based semiconductor microstructures by selective area epitaxy. Journal of Crystal Growth , 310, 1049-1056.

Hoover, W. G. (1985). Canonical dynamics: Equilibrium phase-space distributions. Phys. Rev. A , 31 (3), 1695.

Jones, A., Osowski, M., Lammert, R., Dantzig, J., \& Coleman, J. (1995). Growth, Characterization, and Modeling of Ternary InGaAs-GaAs Quantum Wells by Selective-Area Metalorganic Chemical Vapor Deposition. Journal of ELECTRONIC MATERIALS , 24 (11).

Kiriya, D., Zheng, M., Kapadia, R., Zhang, J., Hettick, M., Yu, Z., et al. (2012). Morphological and spatial control of InP growth using closed-space sublimation. Journal of Applied Physics, 112 (123102).

Lu, F., Tran, T.-T. D., Ko, W. S., N, K. W., Chen, R., \& Chang-Hasnain, C. (2012). Nanolasers grown on silicon-based MOSFETs. OPTICS EXPRESS , 20 (11). 
Noborisaka, J., Motohisa, J., \& Fukui, T. (2005). Catalyst-free growth of GaAs nanowires by selective-area metalorganic vapor-phase epitaxy. Applied Physics Letters , 86 (213102).

Plimpton, S. (19951). Fast Parallel Algorithms for Short-Range Molecular Dynamics. J. Comp. Phys, 117, 1-19.

Ren, F., Ng, K. W., Li, K., Sun, H., \& Chang-Hasnain, C. J. (2013). High-quality InP nanoneedles grown on silicon. Applied Physics Letters, 102.

Stukowski, A. (2010). Visualization and analysis of atomistic simulation data with OVITO - the Open Visualization Tool. Modelling Simul. Mater. Sci. Eng. , 18 (015012).

Swartzentruber, B. S. (1996). Direct Measurement of Surface Diffusion Using Atom-Tracking Scanning Tunneling Microscopy. Physical Review Letters, 76 (3), 459-462.

Terrazas, J., Rodriguez, A., Lopez, C., Escobedo, A., Kuhlmann, F. J., McClure, J., et al. (2005). Ordered polycrystalline thin films for high performance CdTe/CdS solar cells. Thin Solid Films , 490, 146-153.

Ujiharaa, T., Yoshidaa, Y., Leea, W. S., \& Takeda, Y. (2006). Pattern size effect on source supply process for sub-micrometer scale selective area growth by organometallic vapor phase epitaxy. Journal of Crystal Growth , 289, 89-95.

Wang, X., Hartmann, J., Mandl, M., Mohajerani, M. S., Wehmann, H.-H., Strassburg, M., et al. (2014). Growth kinetics and mass transport mechanisms of GaN columns by selective area metal organic vapor phase epitaxy. Journal of Applied Physics, 115 (163104).

Wang, X., Hartmann, J., Mandl, M., Mohajerani, M. S., Wehmann, H.-H., Strassburg, M., et al. (2014). Growth kinetics and mass transport mechanisms of GaN columns by selective area metal organic vapor phase epitaxy. Journal of Applied Physics, 115 (163104).

Wullner, D., Chahoud, M., Schrimpf, T., Bonsch, P., Wehmann, H.-H., \& Schlachetzki, A. (1999). Diffusion during metalorganic vapor-phase epitaxy on V-groove patterned substrates. Journal of Applied Physics, $85(1)$.

Yamaguchi, K., Ogasawara, M., \& Okamoto, K. (1992). Surface-diffusion model in selective metalorganic chemical vapor deposition. Journal of Applied Physics, 72 (12), 5919.

Zhou, X. W., Ward, D. K., Martin, J. E., Swol, F. B., Cruz-Campa, J. L., \& Zubia, D. (2013). Stillinger-Weber potential for the II-VI elements Zn-Cd-Hg-S-Se-Te. Phys. Rev. B , 88 (8), 085309.

Zubia, D., Lopez, C., Rodriguez, M., Escobedo, A., Oyer, S., Romo, L., et al. (2007). Ordered CdTe/CdS Arrays for High-Performance Solar Cells. Journal of ELECTRONIC MATERIALS, 36 (12). 
Table 1. Calculated surface diffusivities for different temperatures and substrate terminations.

\begin{tabular}{|c|c|c|c|c|}
\hline Temperature (K) & Cd on $\mathbf{C d ~}\left(\mathrm{cm}^{\mathbf{2}} / \mathbf{s}\right)$ & Cd on S $\left(\mathrm{cm}^{2} / \mathbf{s}\right)$ & Te on $\mathbf{C d}\left(\mathrm{cm}^{2} / \mathbf{s}\right)$ & Te on S $\left(\mathrm{cm}^{2} / \mathbf{s}\right)$ \\
\hline $\mathbf{6 0 0}$ & $2.0555 \times 10^{-7}$ & $1.7440 \times 10^{-7}$ & $2.6725 \times 10^{-7}$ & $2.1945 \times 10^{-7}$ \\
\hline $\mathbf{8 0 0}$ & $9.6625 \times 10^{-7}$ & $4.6925 \times 10^{-7}$ & $1.6400 \times 10^{-6}$ & $7.1850 \times 10^{-7}$ \\
\hline $\mathbf{1 0 0 0}$ & $2.8350 \times 10^{-6}$ & $3.5925 \times 10^{-7}$ & $2.6725 \times 10^{-6}$ & $1.3995 \times 10^{-6}$ \\
\hline $\mathbf{1 2 0 0}$ & $7.8975 \times 10^{-6}$ & $4.4200 \times 10^{-7}$ & $7.0125 \times 10^{-6}$ & $4.3725 \times 10^{-6}$ \\
\hline $\mathbf{1 4 0 0}$ & $2.6100 \times 10^{-5}$ & $1.7568 \times 10^{-6}$ & $1.6133 \times 10^{-5}$ & $9.3150 \times 10^{-6}$ \\
\hline
\end{tabular}

Table 2. Activation energies $\mathrm{Q}(\mathrm{eV})$ for $\mathrm{Cd}$ and Te surface diffusion on $\mathrm{Cd}$ - and S-terminated CdS.

\begin{tabular}{|c|c|c|c|}
\hline Cd on Cd & Te on Cd & Cd on S & Te on S \\
\hline 0.418 & 0.350 & 0.154 & 0.327 \\
\hline
\end{tabular}


List of Figures

a)

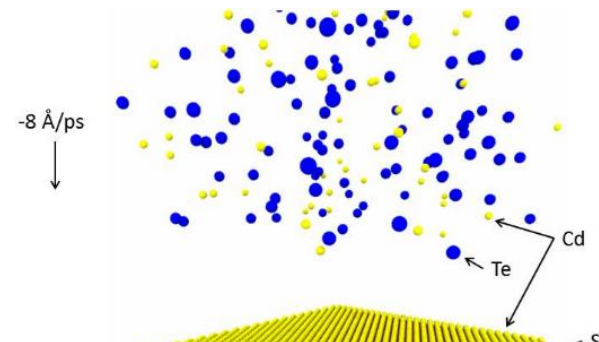

b)
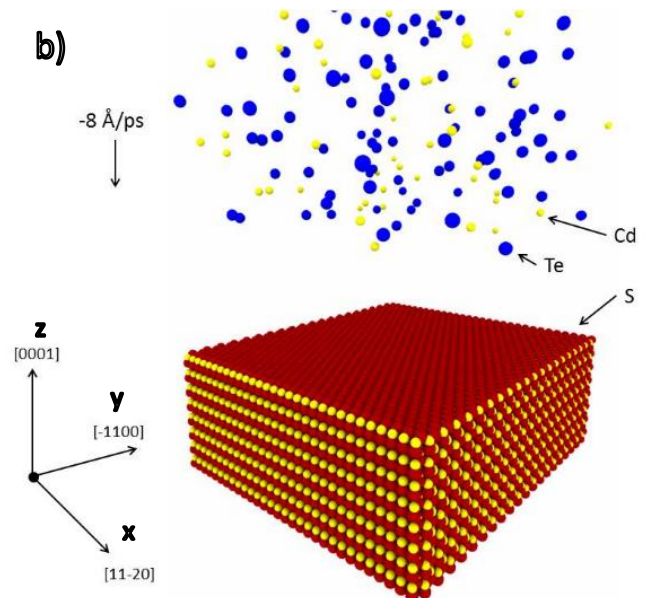

Figure 1. Schematic of the molecular dynamic simulations with a) Cd-terminated and b) Sterminated substrates. Images created with OVITO (Stukowski, 2010).
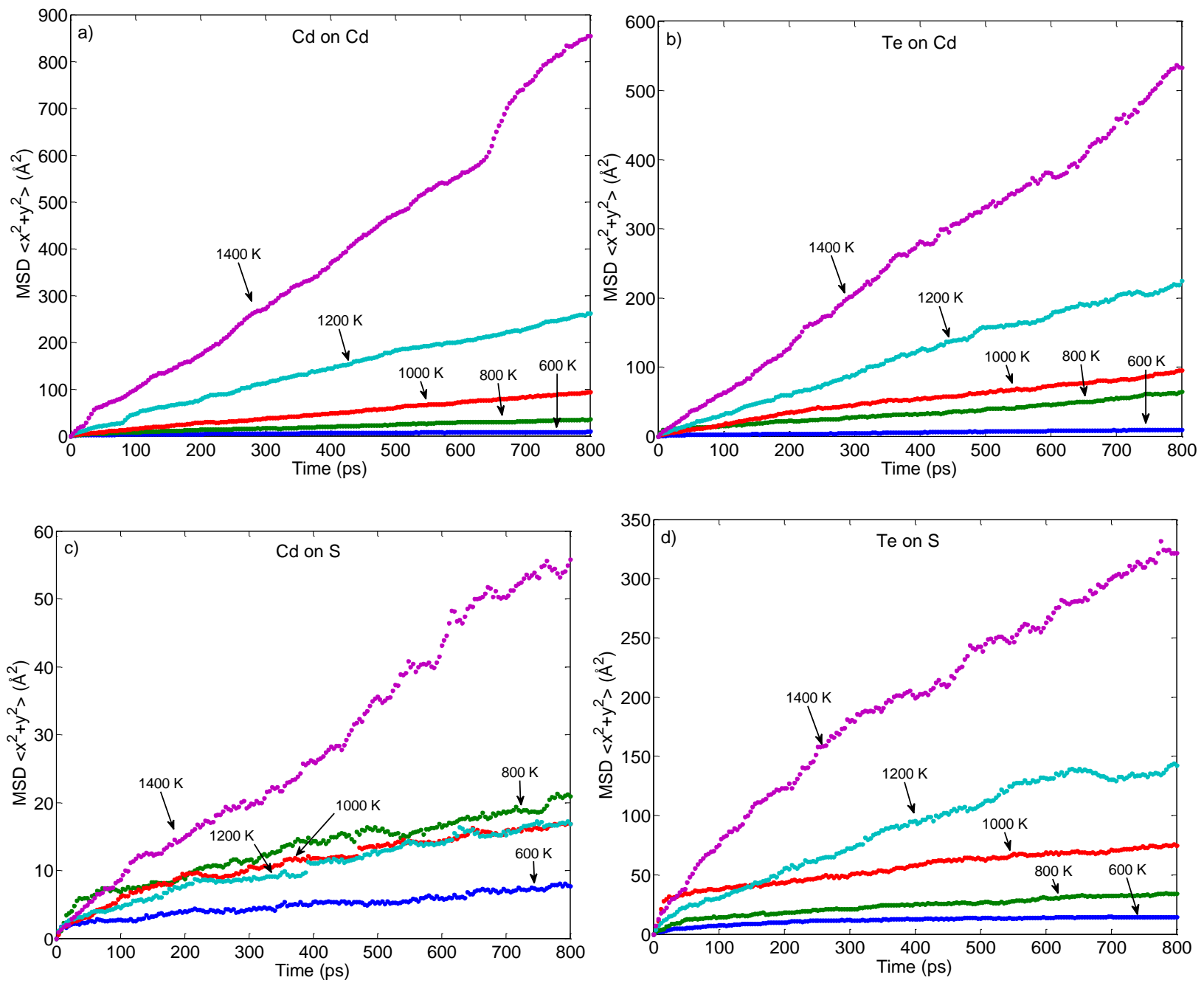
Figure 2. Mean square displacement of adatom on the surface at different temperatures for; (a) $\mathrm{Cd}$ and (b) Te adatoms on Cd-terminated CdS, and (c) Cd and (d) Te adatoms on S-terminated CdS.

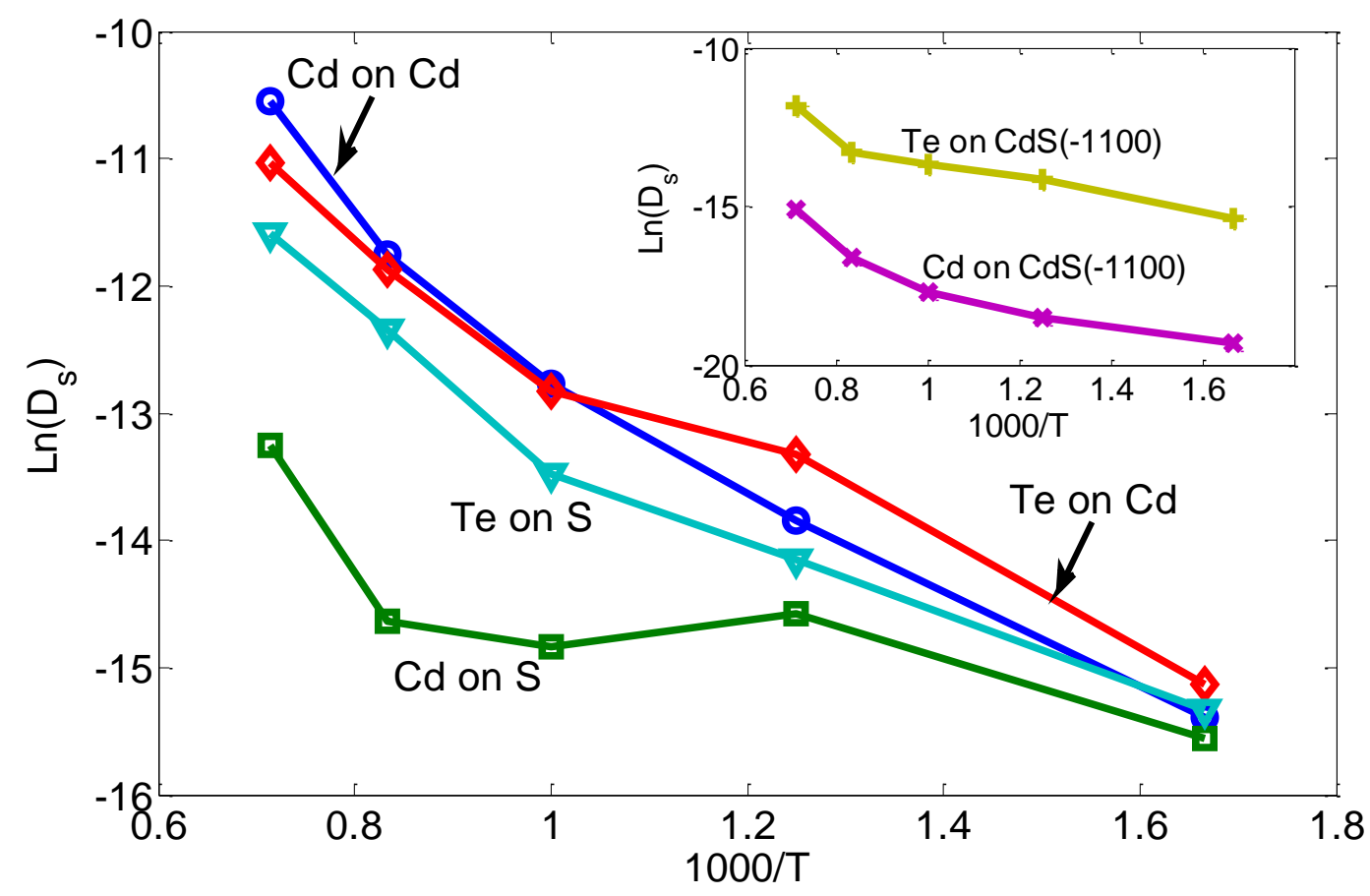

Figure 3. Arrhenius plot for $\mathrm{Cd}$ and Te adatoms on $\mathrm{Cd}$ and $\mathrm{S}$ substrate terminations for $\mathrm{CdS}(0001)$. The inset shows the surface diffusivities for $\mathrm{Cd}$ and Te atoms on CdS(-1100).

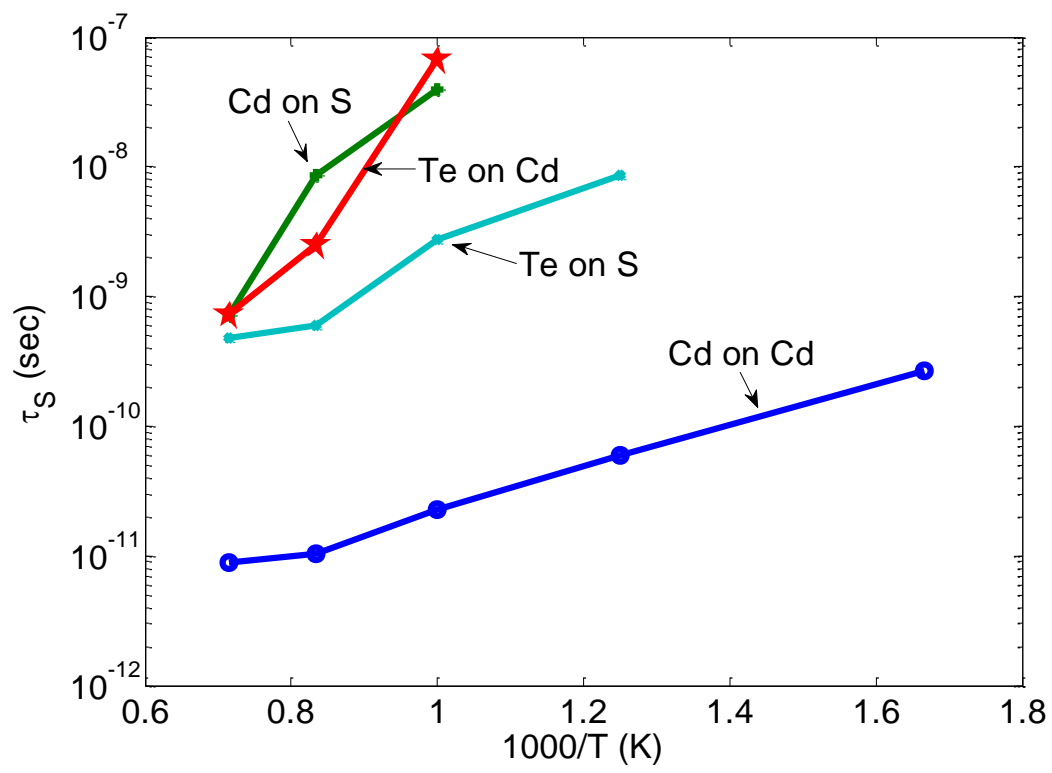

Figure 4. Plot of residence times recorded for all case studies over a range of temperatures. 
a)

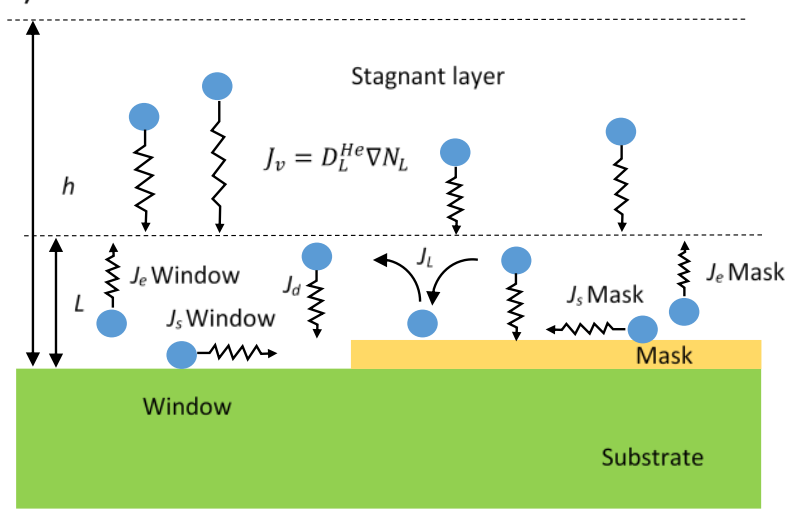

b)

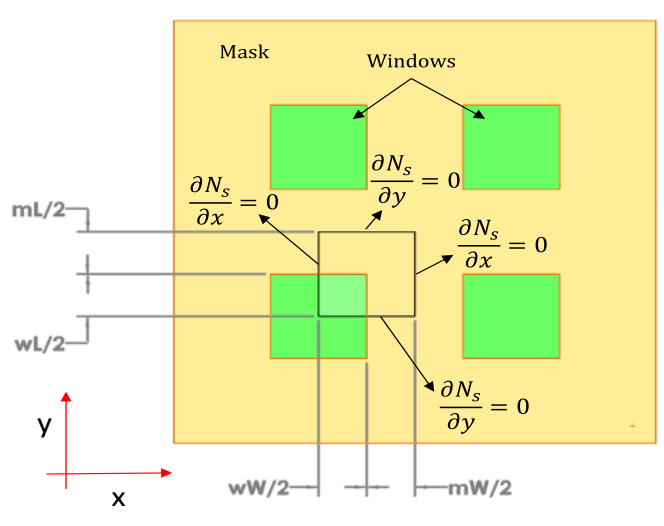

Figure 5. (a) Schematic diagram of the motion of the reactant species in the stagnant layer, $J_{e}$ and $J_{s}$ have distinct values on the mask and window areas. (b) 2D view of the substrate where the continuity equations are solved in the area enclosed by the box under Neumann boundary conditions. $\mathrm{mL}$ indicates mask length, $\mathrm{mW}$ window lengths, $\mathrm{mW}$ mask width, and $\mathrm{wW}$ window width.
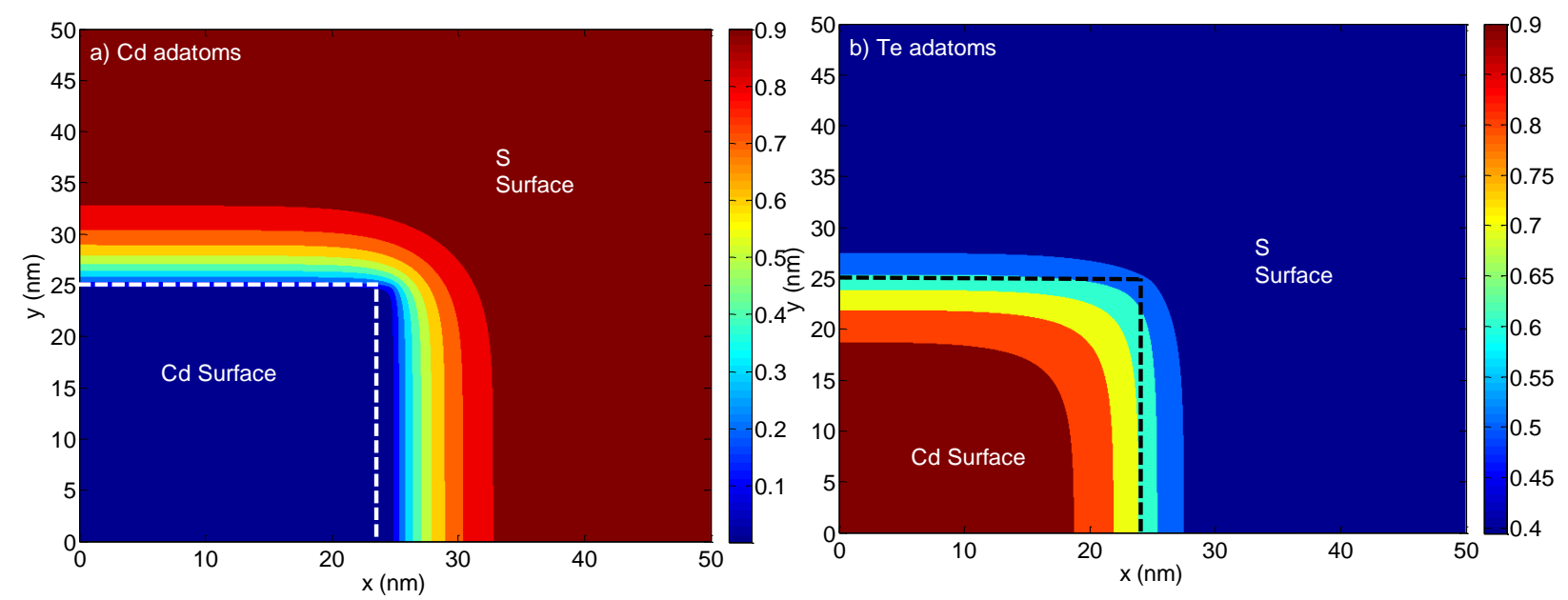

Figure 6. Surface concentration profile for (a) Cd and (b) Te adatoms using the S-terminated substrate as the mask. 